\title{
Enhancement of pDNA Uptake and Expression in H441 Cells by Using PLGA Nanocarriers
}

Cindy Alejandra Gutiérrez-Valenzuela ${ }^{1}$, Pasano Bojang Jr. ${ }^{2}$, Rosalva Josefina Rodríguez-Cordova ${ }^{3}$, Karol Yesenia Hernández-Giottonini ${ }^{3}$, Reynaldo Esquivel ${ }^{3}$, Kenneth S. Ramos ${ }^{2}$, Roberto Guzmán-Z $Z^{4}$, Armando Lucero-Acuña ${ }^{1 *}$

1. Department of Chemical and Metallurgical Engineering, University of Sonora, Hermosillo, MEXICO

2. Department of Medicine, University of Arizona College of Medicine, Tucson, USA

3. Nanotechnology Graduate Program, Department of Physics, University of Sonora, MEXICO

4. Department of Chemical and Environmental Engineering, University of Arizona, Tucson, USA

The use of nanocarriers in medical applications is one of the most promising technologies for drug delivery and vaccination purposes of the last 50 years [1]. In gene therapy, nanocarriers such as polymeric nanoparticles can protect the integrity of plasmid DNA from undesirable systemic interactions. Also, nanocarriers could improve some treatments by controlling the release rate of the encapsulated compound [2-4]. Poly-dl-lactic-co-glycolic acid (PLGA) has been used in the encapsulation of plasmid DNA [5]. One of the advantages of PLGA is that it could contain uncapped carboxylic acids that can be easily functionalized with other molecules before or after nanoparticles preparation [2]. Coupling of fluorescent dyes such as rhodamine can be used to perform in vitro microscopic visualization [6]. In this work, fluoresce labeled polymeric nanoparticles loaded with a plasmid containing the green fluorescent protein gene (pGFP) were prepared by using the double emulsification-solvent evaporation technique. Lung adenocarcinoma cells (H-441) were treated with naked and encapsulated pGFP. Protein expression was compared by observing fluorescence.

Plasmid pGFP used in the encapsulation was replicated using $E$. coli bacteria as a guest in a fermentation process and purified with a kit to obtain a pure plasmid. PLGA coupling to rhodamine B was performed to obtain PLGA-Rh. This polymer modification was verified by FT-IR (results not shown). PLGA-Rh nanoparticles (PNP-Rh), and PLGA-Rh nanoparticles loaded with pGFP (pGFP-PNP) were prepared using double emulsification-solvent evaporation technique [2]. Particles were purified by three cycles of centrifugation $\left(37565^{*} \mathrm{~g}\right.$ for $20 \mathrm{~min}$ ) and freeze-dried. Nanoparticle characterization was performed using a Zetasizer Nano ZS equipment (Malvern) to obtain nanoparticle size distribution and zeta potentials. Surface morphology of pGFP-PNP was analyzed by scanning electron microscopy (SEM) using a JEOL JSM-7800F (JEOL, Pleasanton, CA, USA). In vitro pGFP release from pGFP-PNP was evaluated using the dialysis method [2]. pGFP concentrations were quantified using HPLC-HIC with a source 15PHE column on an Akta purifier 10 UPC (GE Healthcare, Chicago, IL, USA) [2]. H-441 cells were treated with naked plasmid (lipofectamine) and with pGPF-PNP for 24 hours. H-441 cells were imaged live for GFP fluorescence at 40X magnification using Axiovision inverted microscope 48 hours after treatment.

PNP-Rh resulted in $124.5 \pm 1.98 \mathrm{~nm}$ average diameter with polydispersity index (PDI) of $0.047 \pm 0.022$ and displayed zeta potential of $-37.9 \pm 2.40 \mathrm{mV}$. pGFP-PNP size were $163.7 \pm 14.9 \mathrm{~nm}$ with PDI of $0.024 \pm 0.009$ and zeta potential of $-48.8 \pm 5.18 \mathrm{mV}$. The increment in the size and particularly the decrease in zeta potential could reflect the presence of pGFP in the nanoparticle. These results are consistent with other works with encapsulated pDNA [2]. The drug loading resulted in a value of $0.08 \%$ with encapsulation efficiency (EE) of 10.8\%. In vitro release studies for pGFP-PNP were performed (Figure 1), observing a monophasic release over the first 10 days of analysis, suggesting a diffusion mechanism of release. In 
Figure 2, a smooth and spherical surface morphology of pGFP-PNP can be observed. GFP expression in H441 cells transfected with naked plasmid and those incubated with pGFP-PNPs can be appreciated in Figure 3. Rhodamine label spots indicate the location of the nanoparticles, also the spots correspond to the points where green is more intense, confirming successful expression of pGFP. Fluorescent labeled nanoparticles containing plasmid pGFP were effectively prepared. Plasmid was encapsulated successfully without losing integrity or functionality.

References:

[1] J. Kreuter, International Journal of Pharmaceutics 331 (2007), p. 1-10.

[2] C. Gutiérrez-Valenzuela et al., Applied Sciences 6 (2016), p. 364.

[3] F. Danhier et al., Journal of Controlled Release 161 (2012), p. 505-522.

[4] D. Peer et al., Nature Nanotechnology 2 (2007), p. 751-760.

[5] D. Wang et al., Journal of Controlled Release 57 (1999), p. 9-18.

[6] F. Yang et al., Journal of Colloid and Interface Science 302 (2006), p. 159-169.

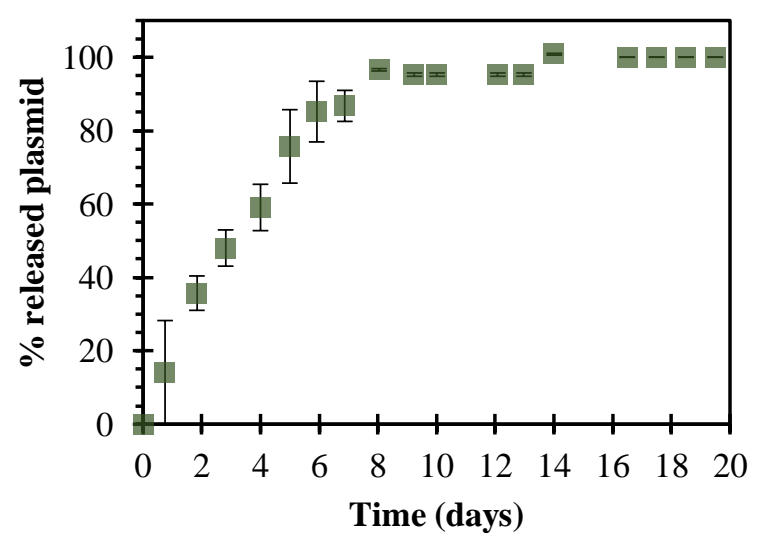

Figure 1. In vitro release kinetics of pGFP-PNP.

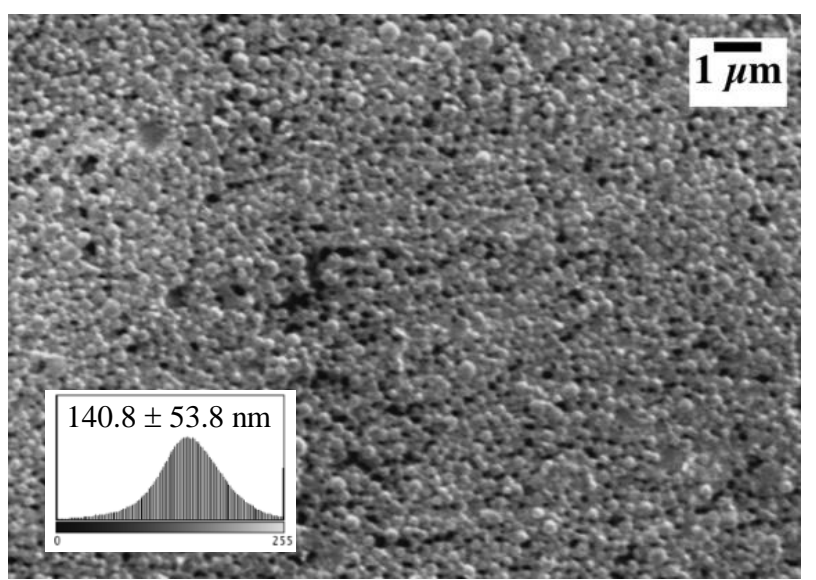

Figure 2. SEM micrograph and histogram of pGFP-PNP.
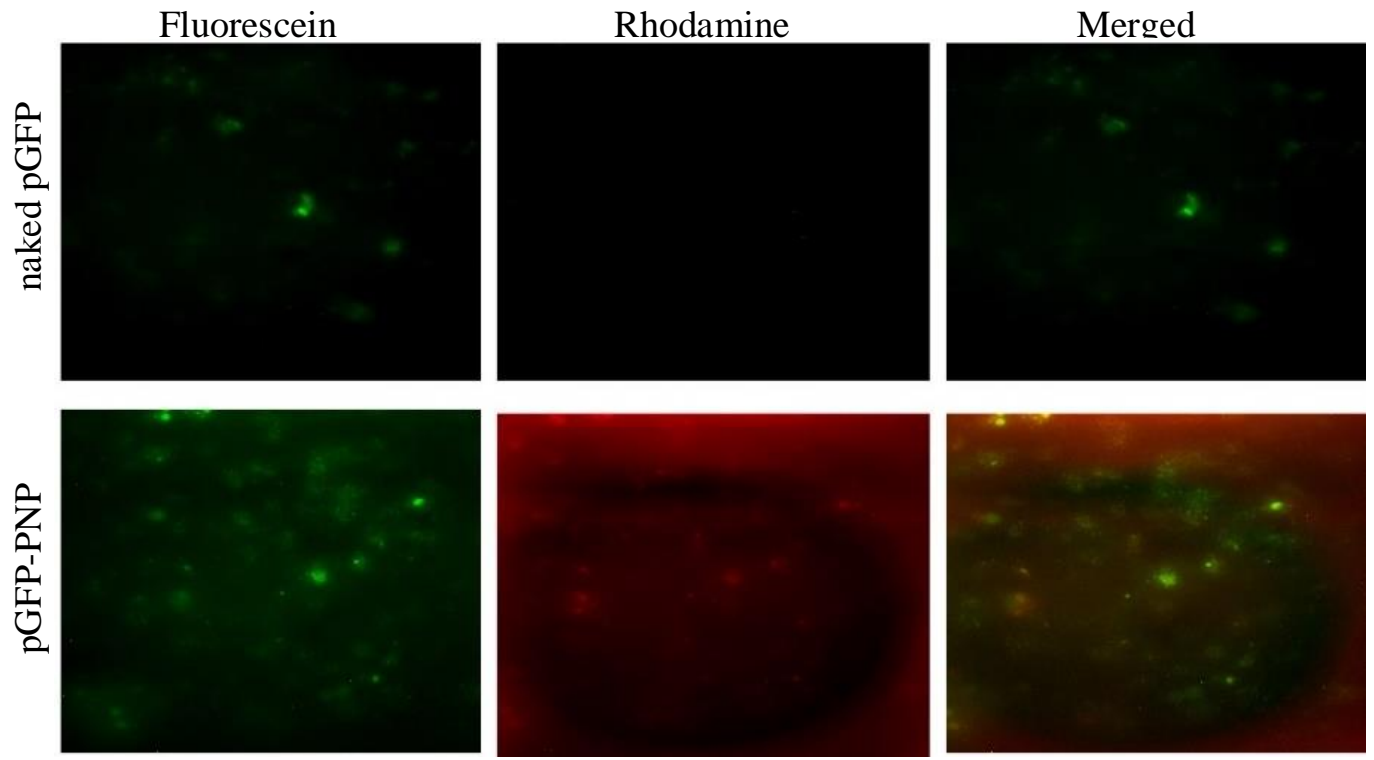

Figure 3. Comparison of expression of naked and encapsulated plasmid in nanoparticles, as well as nanoparticle uptake in H441 cells. 